\title{
Phonon-Induced Thermodynamic Properties of Ultra-narrow Wires
}

\author{
D.I. Ilić ${ }^{a, *}$, J.P. SETRAJČIĆ ${ }^{b}$, And S.K. JAĆIMOVSKI $^{c}$ \\ ${ }^{a}$ University of Novi Sad, Faculty of Technical Sciences, Serbia \\ ${ }^{b}$ University of Novi Sad, Faculty of Sciences, Department of Physics, Serbia \\ ${ }^{c}$ Academy of Criminalistic and Police Studies, Belgrade, Serbia
}

(Received April 1, 2017;in final form December 20, 2017)

\begin{abstract}
In this paper, we investigated the influence of size effect on thermodynamic properties of ultra-narrow wires with a simple cubic lattice, by means of two-time dependent Green functions method, adjusted to confined crystalline structures. Poles of Green functions, which defining phonon spectra, are found by solving the secular equation. For different boundary parameters, this problem is presented graphically. The temperature behavior of ultra-narrow wire thermal capacitance is compared to that of bulk structures. It turned out that in low-temperature region thermal capacitance of the ultra-narrow wire is notably lower than in the corresponding bulk sample. How this fact reflects the thermal, conducting and superconducting properties of materials, is discussed in the conclusion.
\end{abstract}

DOI: 10.12693/APhysPolA.133.57

PACS/topics: Phonons, ultra-narrow wire, the Green function, thermodynamics, specific heat

\section{Introduction}

Over the last few decades, we have experienced a huge improvement in the development of nanoscale devices with physical properties that are dramatically different from the physical characteristics of samples which are larger in size. As a result of numerous factors, nanostructures are characterized by a range of different qualities: superconductive, transport, heat insulating, acoustic and other. Most of these factors are associated with the existence of the boundaries of the structure. In this paper, we will try to observe how dimensional confinement of phonons in low-dimensional structures (ultra-narrow wires) leads to modifications in its thermodynamic properties, which makes the basis for the concept of phonon engineering (nanophononics) [1-4]. Our primary motive is the fact that the influence of size effects on thermal conductivity is becoming extremely important in recent years for heat removal and device design and reliability. The great importance of phonons consists in that without them it is almost impossible to examine and describe the acoustical characteristics, as well as thermodynamic, conductive and superconductive properties of solids.

Ultra-narrow wires [5-9] are crystalline structures with broken translational symmetry in two perpendicular directions ( $z$ and $y$ in our model on Fig. 1), in which conditions on boundaries are different from those inside the structure. Considering that there are no disturbances of the crystalline structure between the boundary surfaces of the wire, we assume it ideal. Otherwise, if there are impurities, vacancies etc, the ultra-narrow wire is deformed. The subject of our research in this paper

*corresponding author; e-mail: dusilic69@uns.ac.rs

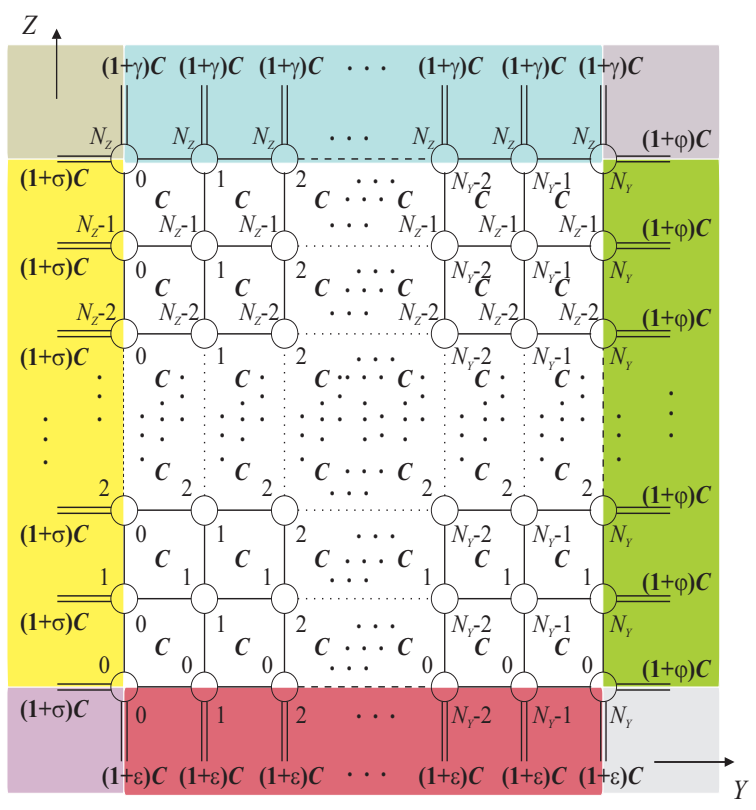

Fig. 1. Cross section (along $X$-plane) of the ultranarrow wire model.

is the ideal ultra-narrow wire of simple cubic crystalline structure, whose basic crystallographic parameters are:

$$
\begin{aligned}
& a_{x}=a_{y}=a_{z}=a ; \quad N_{x} \sim 10^{8} \gg N_{y, z} \sim 10 ; \\
& C_{\boldsymbol{n}, \boldsymbol{m}}^{\alpha, \alpha}=C_{\boldsymbol{n}, \boldsymbol{n} \pm \boldsymbol{\lambda}}^{\alpha} \equiv C_{n_{x} n_{y} n_{z} ; n_{x} \pm 1, n_{y} n_{z}}^{\alpha}= \\
& \quad C_{n_{x} n_{y} n_{z} ; n_{x} n_{y} \pm 1, n_{z}}^{\alpha}=C_{n_{x} n_{y} n_{z} ; n_{x} n_{y} n_{z} \pm 1}^{\alpha} \equiv C^{\alpha} \\
& C_{n_{y} 0 ; n_{y},-1}^{\alpha}=C_{n_{y},-1 ; n_{y} 0}^{\alpha}=(1+\varepsilon) C^{\alpha} ; \\
& C_{n_{y} N_{z} ; n_{y} N_{z}+1}^{\alpha}=C_{n_{y} N_{z}+1 ; n_{y} N_{z}}^{\alpha}=(1+\gamma) C^{\alpha} \\
& C_{0 n_{z} ;-1 n_{z}}^{\alpha}=C_{-1 n_{z} ; 0 n_{z}}^{\alpha}=(1+\sigma) C^{\alpha} ;
\end{aligned}
$$




$$
\begin{aligned}
& C_{N_{y} n_{z} ; N_{y}+1, n_{z}}^{\alpha}=C_{N_{y}+1, n_{z} ; N_{y} n_{z}}^{\alpha}=(1+\varphi) C^{\alpha}, \\
& (\varepsilon, \gamma, \sigma, \varphi) \geq-1,
\end{aligned}
$$

where $n_{y, z} \in\left(0,1,2, \cdots, N_{y, z}\right)$ is the atom site counter along $y$ and $z$ directions. Considering all the above, we can conclude the following about described model structure:

1. Ultra-narrow wires are confined along $y$ and $z$ directions, and unbounded along $x$ direction. That means they have four boundary surfaces: two parallel to the $X Y$ planes (for $z=0$ and $z=L_{z}=N_{z} a$ ), and two parallel to $X Z$ planes (for $y=0$ and $\left.y=L_{y}=N_{y} a\right)$.

2. There are $N_{y}+1$ atoms along $y$-axis, and $N_{z}+1$ atoms along $z$-axis.

3. Torsion Hooke elastic constants $C^{\alpha \beta}$ have much lower values than the straining constants $C_{\alpha}$ so that they can be ignored.

4. Despite the fact that there are no atoms belonging to ultra-narrow wire outside boundary surfaces along $y$ and $z$ directions, we assume that interaction between boundary layers and atoms of external environment nevertheless exists through changed the Hooke forces [8-11]. Elastic constants which describe these interactions are modified with appropriate coefficients $\varepsilon$ and $\gamma, \sigma$ and $\varphi$.

Taking into account the technology of ultra-narrow wires, there is no reason to suppose that it will be on all sides surrounded by a different materials. A simplified, but much more realistic approach (Fig. 2) implies that the atoms that consist the quantum wire are surrounded by atoms belonging to only two different environments $(\sigma, \varphi \rightarrow \varepsilon)$.

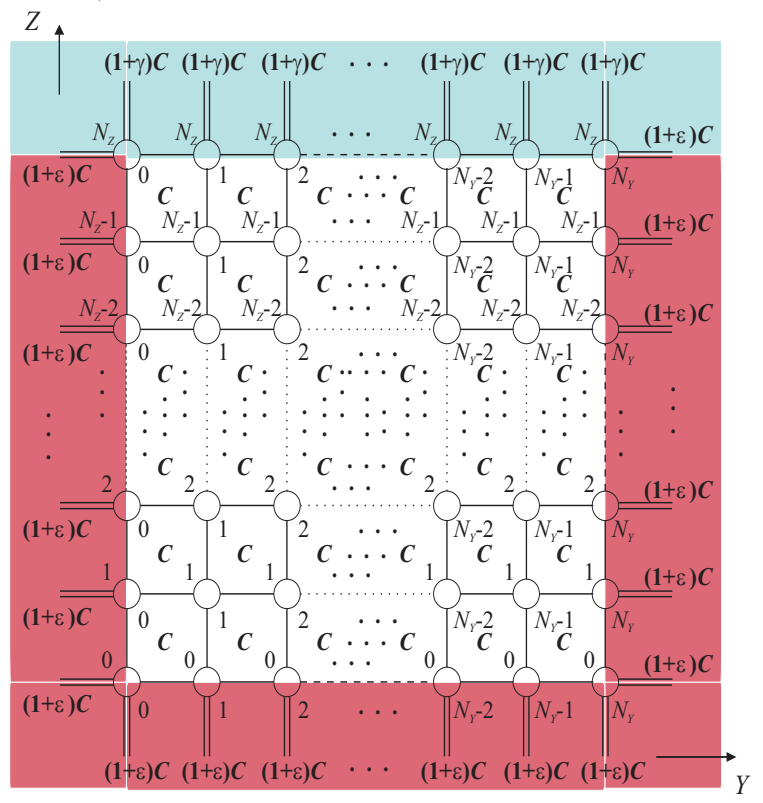

Fig. 2. Realistic model of ultra-narrow wire.
With respect to all these conditions and also regarding the fact that layers with $n_{y} \leq-1$ and $n_{y} \geq N_{y}+1$, and also $n_{z} \leq-1$ and $n_{z} \geq N_{z}+1$ are not present, we have to take into account the following:

$$
\begin{aligned}
& u_{\alpha ; n_{x}, n_{y}, n_{z}}=0 ; \quad-1 \geq n_{y, z} \wedge n_{y, z} \geq N_{y, z}+1 ; \\
& \left(n_{y, z} \notin\left[0, N_{y, z}\right]\right) .
\end{aligned}
$$

These boundary conditions correspond to the free surfaces model, but in reality there are other cases. Another approach often found in the literature is the rigid walls model (or frozen surfaces model), which implies that boundary conditions with zero atomic displacements must be applied on the boundary surfaces. In this paper we have chosen flexible boundary surfaces, considering that this is closer to the real situation in which the ultranarrow wire can "breathe". In contrast, the rigid walls model implies the emergence of phonon standing waves with nodes at the boundaries.

\section{Theoretical analysis}

Our theoretical analysis starts from the standard form of the phonon subsystem Hamiltonian for bulk structures [10-13], written in the harmonic and nearest neighbors approximations. In this case it is adapted to the model structure of ultra-narrow wire presented on Fig. 1, i.e. on Fig. 2:

$$
\begin{aligned}
H & =T+V_{e f f} ; \quad T=\sum_{\alpha ; \boldsymbol{n}} \frac{p_{\alpha ; \boldsymbol{n}}^{2}}{2 M} \\
V_{e f f} & =\sum_{\alpha ; n_{x}, n_{y}, n_{z}} \frac{C_{\alpha}}{4}\left[\left(u_{\alpha ; n_{x}+1, n_{y}, n_{z}}-u_{\alpha ; n_{x}, n_{y}, n_{z}}\right)^{2}\right. \\
& +\left(u_{\alpha ; n_{x}-1, n_{y}, n_{z}}-u_{\alpha ; n_{x}, n_{y}, n_{z}}\right)^{2} \\
& +\left(u_{\alpha ; n_{x}, n_{y}+1, n_{z}}-u_{\alpha ; n_{x}, n_{y}, n_{z}}\right)^{2} \\
& +\left(u_{\alpha ; n_{x}, n_{y}-1, n_{z}}-u_{\alpha ; n_{x}, n_{y}, n_{z}}\right)^{2} \\
& +\left(u_{\alpha ; n_{x}, n_{y}, n_{z}+1}-u_{\alpha ; n_{x}, n_{y}, n_{z}}\right)^{2} \\
& \left.+\left(u_{\alpha ; n_{x}, n_{y}, n_{z}-1}-u_{\alpha ; n_{x}, n_{y}, n_{z}}\right)^{2}\right]
\end{aligned}
$$

Here, $u_{\alpha ; \boldsymbol{n}}$ are the small movements of atom in place $\boldsymbol{n}$ from its equilibrium position in direction $\alpha$, and $p_{\alpha ; \boldsymbol{n}}$ corresponding momentum.

In order to find the phonon dispersion law, we will make use of the phonon two-time commutator Green function $[12-14]$ :

$$
\begin{gathered}
G_{\boldsymbol{n}, \boldsymbol{m}}^{\alpha}\left(t-t^{\prime}\right) \equiv\left\langle\left\langle u_{\alpha ; \boldsymbol{n}}(t) \mid u_{\alpha ; \boldsymbol{m}}\left(t^{\prime}\right)\right\rangle\right\rangle= \\
\Theta\left(t-t^{\prime}\right)\left\langle\left[u_{\alpha ; \boldsymbol{n}}(t), u_{\alpha ; \boldsymbol{m}}\left(t^{\prime}\right)\right]\right\rangle_{0}
\end{gathered}
$$

and corresponding equation of motion:

$$
\begin{aligned}
& -M \omega^{2} G_{\boldsymbol{n}, \boldsymbol{m}}^{\alpha}(\omega)= \\
& \quad-\frac{\mathrm{i} \hbar}{2 \pi} \delta_{\boldsymbol{n}, \boldsymbol{m}}+\frac{1}{\mathrm{i} \hbar}\left\langle\left\langle\left[p_{\alpha ; \boldsymbol{n}}, H\right] \mid u_{\alpha ; \boldsymbol{m}}\right\rangle\right\rangle_{\omega} .
\end{aligned}
$$

The next step consists in calculating the commutators in Green function from equation (4). By applying the partial spatial Fourier-transformation along $y$ and $z$ directions, where the translational symmetry has been 
disrupted:

$$
\begin{aligned}
& G_{n_{x} n_{y} n_{z} ; \boldsymbol{m}}^{\alpha}(\omega)= \\
& \quad \frac{1}{N_{x}} \sum_{k_{x}} \mathrm{e}^{\mathrm{i} k_{x} a\left(n_{x}-m_{x}\right)} G_{n_{y} n_{z} ; m_{y} m_{z}}^{\alpha}\left(k_{x} ; \omega\right) ; \\
& \delta_{n_{x} m_{x}} \delta_{n_{y} m_{y}} \delta_{n_{z} m_{z}}= \\
& \quad \frac{1}{N_{x}} \sum_{k_{x}} \mathrm{e}^{\mathrm{i} k_{x} a\left(n_{x}-m_{x}\right)} \delta_{n_{y} m_{y}} \delta_{n_{z} m_{z}},
\end{aligned}
$$

we get the system of $\left(N_{y}+1\right) \times\left(N_{z}+1\right)$ nonhomogeneous algebraic-difference equations with the same number of undetermined Green functions [10-13]:

$$
\begin{gathered}
G_{n_{y}-1, n_{z}} \\
\mathcal{K}_{n_{y} n_{z}}=+G_{n_{y}, n_{z}-1}+\varrho G_{n_{y}, n_{z}}+G_{n_{y}, n_{z}+1}, \\
+G_{n_{y}+1, n_{z}}
\end{gathered}
$$

where:

$$
\begin{aligned}
& \varrho=\frac{\omega^{2}}{\Omega_{\alpha}^{2}}-4\left(1+\sin ^{2} \frac{a k_{x}}{2}\right) ; \\
& G_{n_{y}, n_{z}} \equiv G_{n_{y} n_{z} ; m_{y} m_{z}}^{\alpha}\left(k_{x} ; \omega\right) ; \\
& \Omega_{\alpha}^{2}=\frac{C_{\alpha}}{M} ; \mathcal{K}_{n_{y}^{\prime \prime} n_{z}}^{\prime \prime}=\frac{\mathrm{i} \hbar}{2 \pi C_{\alpha}} \delta_{n_{y} n_{z}, m_{y} m_{z}} .
\end{aligned}
$$

Next, we have to determine the zeros of the determinant associated with the system of equations (6), which - generally speaking - is not an analytically solvable task. It can be performed numerically, for given values of parameters $\varepsilon, \gamma, N_{y}$ and $N_{z}$. Therefore, we will focus on the model of the free surfaces [10,11], when surface perturbations are so small, that can be neglected $(\varepsilon=\gamma=0)$. This means that the Hooke interaction of surface atoms of ultra-narrow wire with atoms/molecules of surrounding environments is of the same type and intensity. We called this model "a model of ideal quantum wire with free surfaces". It is particularly interesting because an analytical solution for the phonon dispersion law and other physical characteristics exists. This choice of model favors the quantum size effect in determining the microscopic and macroscopic properties of the sample $[1,9]$. The contributions of all other confinement effects (shape, etc) are negligible and can only slightly affect changes caused by the size effect $[2,3,5-7]$.

The Green functions from equation (6) can be expressed in form:

$$
G_{n_{y}, n_{z}}=\frac{\mathcal{D}_{n_{y}, n_{z}}}{\mathcal{D}_{N_{y}+1, N_{z}+1}},
$$

where $\mathcal{D}_{n_{y}, n_{z}}$ is the variable-determinant and $\mathcal{D}_{N_{y}+1, N_{z}+1}$ the two-dimensional determinant of the system. Poles of Green functions can be defined by the equation: $\mathcal{D}_{N_{y}+1, N_{z}+1} \equiv 0$, and determinant $\mathcal{D}_{N_{y}+1, N_{z}+1}$ can be determined through the Chebyshev polynomials of second order. In that manner, we get the dispersion law of phonons in form:

$$
\begin{gathered}
\mathcal{E}_{\boldsymbol{k}}^{\alpha}=2 \sqrt{\sin ^{2} \frac{a k_{x}}{2}+\sin ^{2} \frac{a k_{y}(\mu)}{2}+\sin ^{2} \frac{a k_{z}(\nu)}{2}} \\
\left(\mu=1,2, \ldots, N_{y}+1 ; \nu=1,2, \ldots, N_{z}+1\right), \text { which is }
\end{gathered}
$$

almost exactly the same as the one for bulk structures. The difference between the two equations is, however, that quasimomentum of phonons in ultra-narrow wires can take only discrete values in $y$ and $z$ directions, and is continual in $x$ direction. It also can be shown that minimum phonon energy in ultra-narrow wires is not equal to zero:

$$
\begin{aligned}
& \Delta \equiv \Delta_{\min }=\mathcal{E}_{\boldsymbol{k}_{\min }}^{\alpha}=\mathcal{E}_{k_{x}=0, k_{y}=k_{y}^{\min }, k_{z}=k_{z}^{\min }}^{\alpha}= \\
& 2 \sqrt{\sin ^{2} \frac{a k_{y}^{\min }(\mu)}{2}+\sin ^{2} \frac{a k_{z}^{\min }(\nu)}{2}}, \\
& k_{y}^{\min }(\mu)=k_{y}^{\min }(1)=\frac{\pi}{a} \frac{1}{N_{y}+2} ; \\
& k_{z}^{\min }(\nu)=k_{z}^{\min }(1)=\frac{\pi}{a} \frac{1}{N_{z}+2},
\end{aligned}
$$

and the corresponding minimum phonon frequency is:

$$
\begin{aligned}
\omega_{\alpha}^{\min } & =2 \frac{v}{a} \\
& \times \sqrt{\sin ^{2} \frac{\pi}{2\left(N_{y}+2\right)}+\sin ^{2} \frac{\pi}{2\left(N_{z}+2\right)}} \neq 0 .
\end{aligned}
$$

The Fig. 3 graphically shows the spectra of phonons in ultra-narrow wires $\mathcal{E}=\mathcal{E}_{\mu, \nu}\left(X^{2}\right)$, where $X \equiv \sin \left(a k_{x} / 2\right)$ for $\mu=5$ and $\nu=4$. The blue-colored dashed lines limit the continual zone of allowed phonon energies in bulk structures. Energy gaps and discreteness of phonon energy, which are the main consequences of spatial confinement, also can be seen.

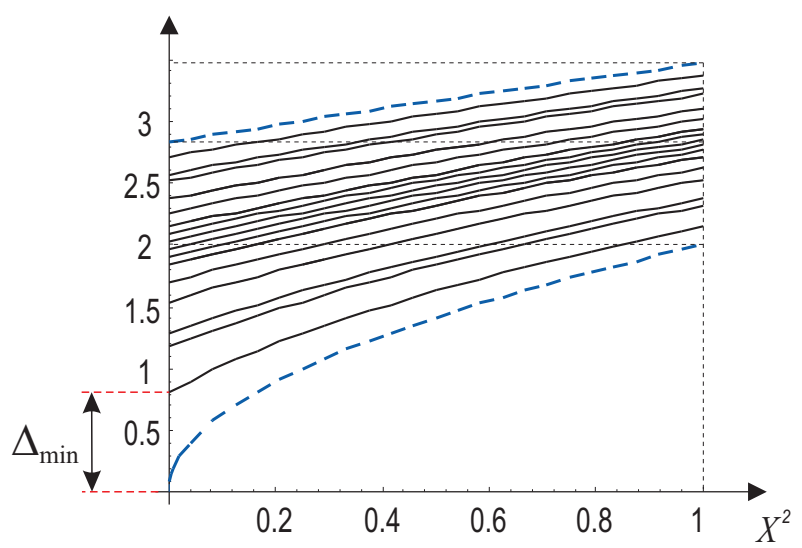

\section{Fig. 3hermod spectra in ultrannarrow wires.}

Our main goal in this paper is to determine the physical quantities that define the thermodynamic properties of ultra-narrow wire, in order to compare them with those of larger samples. To this end, it is necessary first to determine corresponding values for the Debye wave vector $\left(k_{D}^{w}\right)$ and the Debye frequency $\left(\omega_{D}^{w}\right)$. Assuming that the wave vectors of phonons in ultra-narrow wire are distributed within a sphere of radius $k_{D}^{w}$, their possible values along $x, y$, and $z$ directions are:

$$
k_{x} \in\left[-\frac{\pi}{a},+\frac{\pi}{a}\right] \Rightarrow \Delta k_{x}=\frac{2 \pi}{a},
$$




$$
\begin{gathered}
k_{y / z} \in\left[\frac{1}{N_{y / z}+2} \cdot \frac{\pi}{a}, \frac{N_{y / z}+1}{N_{y / z}+2} \cdot \frac{\pi}{a}\right] \Rightarrow \\
\Delta k_{y / z}=\frac{N_{y / z}}{N_{y / z}+2} \cdot \frac{\pi}{a},
\end{gathered}
$$

from which it follows:

$$
\begin{gathered}
V=\frac{4}{3} \pi\left(a k_{D}^{w}\right)^{3} a^{3} \Delta k_{x} \Delta k_{y} \Delta k_{z} \Rightarrow \\
k_{D}^{w}=\sqrt[3]{\frac{3 \pi^{2}}{2 a^{3}}} \cdot \sqrt[3]{\frac{N_{y}}{N_{y}+2} \cdot \frac{N_{z}}{N_{z}+2}} \Rightarrow
\end{gathered}
$$

$$
\begin{aligned}
& k_{D}^{w}=k_{D}^{b} \cdot \sqrt[3]{\frac{N_{y}}{2\left(N_{y}+2\right)} \cdot \frac{N_{z}}{2\left(N_{z}+2\right)}}= \\
& k_{D}^{f} \cdot \sqrt[3]{\frac{N_{y}}{2\left(N_{y}+2\right)}},
\end{aligned}
$$

where $k_{D}^{b}=\sqrt[3]{6 \pi^{2}} / a$ is the Debye wave vector for bulk. In order to obtain the number of allowed values of $\boldsymbol{k}$ per volume unit of $\boldsymbol{k}$-space, we are using the next adjusted expression:

$$
\mathcal{D}_{D}^{w}(\omega)=\frac{3 N_{x} N_{y}^{w} N_{z}^{w} a^{3}}{(2 \pi)^{3}} \int_{0}^{\pi} \sin \Theta \mathrm{d} \Theta \int_{0}^{2 \pi} \mathrm{d} \varphi \int_{k_{\min }}^{k_{D}} k^{2} \mathrm{~d} k \delta(\omega-v k)=\frac{N_{x} N_{y}^{w} N_{z}^{w} a^{3}}{2 \pi^{2}} \cdot \frac{\omega^{2}}{v^{3}} .
$$

In the next step, we are applying the normalization condition: starting from the fact that the total number of phonon states is equal to the number of atoms, we get

$$
\int_{\omega_{\min }}^{\omega_{D}} \mathcal{D}_{D}^{w}(\omega) \mathrm{d} \omega=N \Rightarrow \frac{N_{x} N_{y}^{w} N_{z}^{w} a^{3}}{2 \pi^{2} v^{3}} \int_{\omega_{\min }}^{\omega_{D}} \omega^{2} \mathrm{~d} \omega=N_{x}\left(N_{y}^{w}+1\right)\left(N_{z}^{w}+1\right),
$$

and, as a result, Debye frequency in ultra-narrow wire is obtained in form:

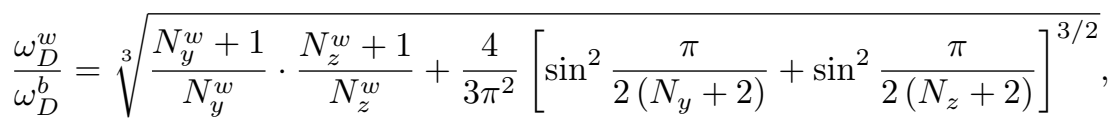

where: $\omega_{D}^{b}=k_{D}^{b} \cdot v$ is Debye frequency in corresponding unbounded structure. By analyzing equation (12) it can be concluded that Debye frequency has somewhat higher value in ultra-narrow wire, than in sample that is larger in size.

Ultra-narrow wire internal energy is calculated by means of standard definitional form:

$$
U_{w}=\int \mathrm{d} \omega^{w} \mathcal{D}\left(\omega^{w}\right)\left\langle n\left(\omega^{w}, T\right)\right\rangle \hbar \omega^{w}=\int_{\omega_{\min }^{w}}^{\omega_{D}^{w}} \mathrm{~d} \omega^{w}\left(\frac{\left(\omega^{w}\right)^{2} V}{2 \pi^{2} v^{3}}\right)\left(\frac{\hbar \omega^{w}}{\mathrm{e}^{\frac{\hbar \omega_{\mathrm{B}}}{k^{T}}}-1}\right)=9 N_{w} k_{\mathrm{B}} T\left(\frac{T}{T_{D}^{w}}\right)^{3} \int_{x_{\min }^{w}}^{x_{D}^{w}} \frac{\left(x^{w}\right)^{3}}{\mathrm{e}^{x^{w}}-1} \mathrm{~d} x^{w},(13
$$

where: $N_{w}=N_{x}\left(N_{y}+1\right)\left(N_{z}+1\right), x^{w}=\frac{\hbar \omega^{w}}{k_{\mathrm{B}} T}, x_{D}^{w}=\frac{T_{D}^{w}}{T}, T_{D}^{w}=\frac{\hbar \omega_{D}^{w}}{k_{\mathrm{B}}}$ and $x_{\min }^{w}=\frac{\hbar \omega_{\min }^{w}}{k_{\mathrm{B}} T}$.

In low temperature region $\left(x_{D}^{w} \rightarrow \infty\right)$, equation (13) becomes:

$$
U_{w}=9 N_{w} k_{\mathrm{B}} T\left(\frac{T}{T_{D}^{w}}\right)^{3} \int_{x_{\min }^{w}}^{\infty} \frac{\left(x^{w}\right)^{3}}{\mathrm{e}^{x^{w}}-1} \mathrm{~d} x^{w}=9 N_{w} k_{\mathrm{B}} T\left(\frac{T}{T_{D}^{w}}\right)^{3}\left[\frac{\pi^{4}}{15}-\int_{0}^{x_{\min }^{w}} \frac{\left(x^{w}\right)^{3}}{\mathrm{e}^{x^{w}}-1} \mathrm{~d} x^{w}\right],
$$

i.e, taking into account the series expansion $\left(\mathrm{e}^{t}-1\right)^{-1}=\sum_{j=1}^{\infty} \mathrm{e}^{-j t}$ :

$$
U_{w}=9 N_{w} k_{\mathrm{B}} \frac{T^{4}}{\left(T_{D}^{w}\right)^{3}}\left\{\frac{\pi^{4}}{15}+\sum_{j=1}^{\infty} \frac{1}{j} \mathrm{e}^{-j x_{\min }^{w}}\left[\left(x_{\min }^{w}\right)^{3}+\frac{3}{j}\left(x_{\min }^{w}\right)^{2}+\frac{6}{j^{2}} x_{\min }^{w}+\frac{6}{j^{3}}\right]-\sum_{j=1}^{\infty} \frac{6}{j^{4}}\right\} .
$$

In order to find the ultra-narrow wire thermal capacitance per an unit cell (i.e, per an atom), we used the standard equation $C_{w}^{*}=\frac{1}{N_{w}} \frac{\partial U_{w}}{\partial T}$ and we obtain:

$$
\begin{aligned}
& C_{w}^{*}=\frac{12 \pi^{4}}{5} k_{\mathrm{B}}\left(\frac{T}{T_{D}^{w}}\right)^{3} \\
& \quad+9 k_{\mathrm{B}}\left(\frac{T}{T_{D}^{w}}\right)^{3} \sum_{j=1}^{\infty} \frac{1}{j} \mathrm{e}^{-j x_{\min }^{w}}\left[\frac{1}{j}\left(x_{\min }^{w}\right)^{4}+\left(1+\frac{3}{j^{2}}\right)\left(x_{\min }^{w}\right)^{3}+\frac{6}{j}\left(1+\frac{1}{j^{2}}\right)\left(x_{\min }^{w}\right)^{2}+\frac{6}{j^{2}}\left(3+\frac{1}{j^{2}}\right) x_{\min }^{w}+\frac{24}{j^{3}}\right] .
\end{aligned}
$$

By introducing the following marks: $\frac{T}{T_{D}^{b}} \equiv \mathcal{T} ; \frac{\omega_{\min }^{w}}{\omega_{D}^{b}} \equiv \Delta_{w}$;

$$
\frac{\omega_{D}^{w}}{\omega_{D}^{b}}=\left\{\frac{N_{y}^{w}+1}{N_{y}^{w}} \cdot \frac{N_{z}^{w}+1}{N_{z}^{w}}+\frac{4}{3 \pi^{2}}\left[\sin ^{2} \frac{\pi}{2\left(N_{y}+2\right)}+\sin ^{2} \frac{\pi}{2\left(N_{z}+2\right)}\right]^{3 / 2}\right\}^{1 / 3} \equiv f\left(N_{y}, N_{z}\right),
$$


it follows:

$$
\frac{T}{T_{D}^{w}}=\frac{\omega_{D}^{b}}{\omega_{D}^{w}} \cdot \mathcal{T}=\frac{\mathcal{T}}{f\left(N_{y}, N_{z}\right)}, \quad x_{\min }^{w}=\frac{T_{D}^{w}}{T} \cdot \frac{\omega_{\min }^{w}}{\omega_{D}^{w}}=\frac{\Delta_{w}}{\mathcal{T}},
$$

previous equation becomes:

$$
\begin{aligned}
C_{w}^{*} & =\frac{9 k_{\mathrm{B}}}{f^{3}\left(N_{y}, N_{z}\right)} \\
& \times\left\{\frac{4 \pi^{4}}{15} \mathcal{T}^{3}+\sum_{j=1}^{\infty} \frac{1}{j} \mathrm{e}^{-j \frac{\Delta_{w}}{\mathcal{T}}} \cdot\left[\frac{1}{j} \frac{\Delta_{w}^{4}}{\mathcal{T}}+\left(1+\frac{3}{j^{2}}\right) \Delta_{w}^{3}+\frac{6}{j}\left(1+\frac{1}{j^{2}}\right) \Delta_{w}^{2} \mathcal{T}+\frac{6}{j^{2}}\left(3+\frac{1}{j^{2}}\right) \Delta_{w} \mathcal{T}^{2}+\frac{24}{j^{3}} \mathcal{T}^{3}\right]\right\} .
\end{aligned}
$$

In order to compare this expression with the one obtained for the unbounded crystalline structures, we divided equation (16) by the constant: $C_{0}=\frac{12 \pi^{4}}{5} k_{\mathrm{B}}$, whose dimension is equal to dimension of thermal capacitance:

$$
\begin{aligned}
\tilde{C_{w}} & \equiv \frac{C_{w}^{*}}{C_{0}}=f^{-3}\left(N_{y}, N_{z}\right) \\
& \times\left\{\mathcal{T}^{3}+\frac{15}{4 \pi^{4}} \sum_{j=1}^{\infty} \frac{1}{j} \mathrm{e}^{-j \frac{\Delta_{w}}{\mathcal{T}}}\left[\frac{1}{j} \frac{\Delta_{w}^{4}}{\mathcal{T}}+\left(1+\frac{3}{j^{2}}\right) \Delta_{w}^{3}+\frac{6}{j}\left(1+\frac{1}{j^{2}}\right) \Delta_{w}^{2} \mathcal{T}+\frac{6}{j^{2}}\left(3+\frac{1}{j^{2}}\right) \Delta_{w} \mathcal{T}^{2}+\frac{24}{j^{3}} \mathcal{T}^{3}\right]\right\} .
\end{aligned}
$$

Calculation of phonon contribution in thermodynamic properties of low-dimensional structures (ultra-thin film and superlattice) was the subject of our previous investigations and has been referred $[4,10,11,15-20]$ in the past period. Here, we will use some of these results in order to compare them with the results obtained in this paper for ultra-narrow wires.

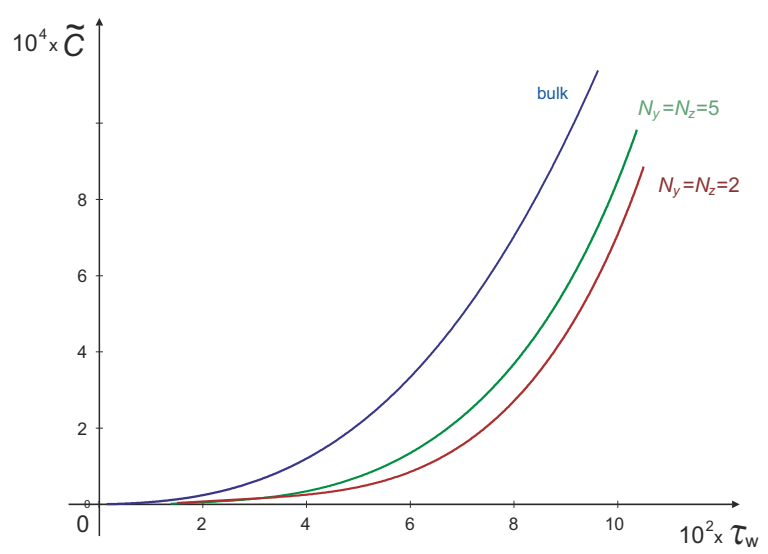

Fig. 4. Low-temperature behavior of thermal capacitance.

Figure 4 shows relative thermal capacitances $(\tilde{C})$ of bulk sample and ultra-narrow wire subject to the relative temperature $(\mathcal{T})$ for $N_{y}=N_{z}=2$ and $N_{y}=N_{z}=5$ in low-temperature region. In order to obtain the required dependency in graphical form, we selected the following parameter values:

- the speed of sound in the crystal: $v \approx 10^{4} \mathrm{~m} / \mathrm{s}$;

- lattice constant: $a \approx 10^{-10} \mathrm{~m}$;

- Debye frequency in bulk: $\omega_{D}^{b} \approx 4 \cdot 10^{14} \mathrm{rad} / \mathrm{s}$;
- minimum phonon frequency in ultra-narrow wire (for $N_{y}=N_{z}=2$ ): $\omega_{\min }^{w} \approx 1.0824 \cdot 10^{14} \mathrm{rad} / \mathrm{s}$.

The figure shows that thermal capacitance of confined crystalline structure (ultra-narrow wire) is considerably lower than that of massive samples. It is also evident that with an increase in a number of layers thermal capacitance of ultra-narrow wire tends toward that of bulk structure.

\section{Conclusions}

Understanding the basic physical properties of nanostructures is essential for their implementation. Thermodynamic characteristics related to phonon movements through the nanometer-sized samples are of particular interest. Independent of the type of lattices, the thermodynamics of their subsystems (electrons, magnons, excitons, solitons, etc.) is determined when the subsystem is in thermodynamic equilibrium with phonons. Phonons are collective mechanical oscillations of atoms and represent the most important system of excitations, because they are present in all systems and their influence, more or less, changes behavior of all other objects or excitations of the system. In this paper, we applied a rigid theoretical approach that implies that the ultra-narrow wire is surrounded by different materials from every side. In that manner, desirable properties of the structure can be changed by changing the lattice constant, by inserting atoms of different kinds and by changing the parameters $\varepsilon, \gamma, \sigma$ and $\varphi$. However, taking into account the production techniques of ultra-narrow wire, there is no reason to suppose that it will be on all sides surrounded by a different materials. A simplified, but much more realistic approach implies that the atoms that consist the ultranarrow wire are surrounded by atoms belonging to only two different environments $(\sigma, \varphi \rightarrow \varepsilon)$.

Temperature analysis of phonon contribution to the thermodynamic characteristics behavior of ultra-narrow 
wire was carried out in detail, and - with respect to all of the above mentioned - we came to the following conclusions: it is well known fact that the phonons at Debye frequencies largely affect the transport properties of materials [12-14]. In this paper, we have chosen the model of ideal quantum wire with free surfaces $(\varepsilon=\gamma=0)$, because only this kind of model enables us to obtain analytical solutions and encompasses all the impacts of the quantum size effect on its thermodynamic properties.

Our results, therefore, suggest that the ultra-narrow wire will be inferior electrical and thermic conductor in comparison to the structures that are larger in size, with no chemical and structural differences between them. But it is also established that poor conductors (under normal conditions) are at the same time the best superconductors, and vice versa [4,15-18,21-23]. Against this background, it can be concluded that in the spatially very limited structures the best superconducting properties can be expected, or that restriction of the spatial dimensions of the structure plays a major role in improving superconducting properties of material.

Finally, it should be noted that the results obtained are not completely conclusive, because the study included ideal structures with extremely regular geometrical shapes and surfaces, free from defects that real samples are required to have. On the other hand, the thermal behavior of materials are affected by many different factors: the density, thermal expansion of the material, the purity of the sample, surface absorption, sample size, etc, whereby for each particular material the different effect is dominant. Only with careful and complete theoretical and experimental analysis of individual properties of nanostructures can be investigated thermodynamic behavior of materials. For this purpose it is necessary to provide many more theoretical calculations and develop different methods for their determination.

\section{Acknowledgments}

This paper was in part supported by the Serbian Ministry of Education, Science and Technological Development (Grants: ON-171039 and TR-34019) and Ministry of Science and Technology of the Republic of Srpska (Grants: 19/6-020/961-15/16) as well as the Vojvodina Provincial Secretariat for Higher Education and Scientific-Research Development (Grant: 142-451$2469 / 2017-01 / 02)$.

\section{References}

[1] A.A. Balandin, D.L. Nika, Materials Today 15, 266 (2012).

[2] A.A. Balandin, Moldavian Journal of the Physical Sciences 6, 1 (2007).
[3] N. Yang, X. Xu, G. Zhang, B. Li, AIP Advances 2, 041410 (2012).

[4] J.P. Šetrajčić, S.K. Jaćimovski, V.D. Sajfert, Modern Physics Letters B 29, 1550008 (2015).

[5] P. Harrison, Quantum Wells, Wires, Dots, John Wiley\&Sons, Chichester 2005.

[6] C.N.R.Rao, A.Govindaraj, Nanotubes, Nanowires, RSC Publishing, Cambridge 2005.

[7] M.A. Reed, W.P. Kirk, Nanostructure Physics and; Fabrication, Eds.: M.A. Reed, W.P. Kirk, Academic Press, Boston 1989.

ISBN: 9780323138956

[8] M.G. Cottam, D.R. Tilley, Introduction to Surface, Superlattice Excitations, University Press, Cambridge 1989.

[9] S.G. Davison, M. Steslicka, Basic Theory of Surface; States, Clarendon, Oxford 1996.

ISBN: 9780198518969

[10] S.K. Jaćimovski, D.I. Ilić, I.K. Junger, J.P. Šetrajčić, Novi Sad J. Math. 31, 55 (2001).

[11] J.P. Šetrajčić, S.K. Jaćimovski, D. Raković, D.I. Ilić, Phonon Spectra in Crystalline Nanostructures, in: Electrical, Computer Engineering Series, pp. 146; Advances in Simulation, Systems Theory, Systems Engineering, Eds.: N.E. Mastorakis, V.V. Kluev, Dj. Koruga, WSEAS Press, Singapore 2002.

[12] C. Kittel, Introduction to Solid State Physics, Wiley,; New York 1986.

ISBN 978-0-471-41526-8

[13] G. Rickayzen, Green Functions and Condensed Matter, Academic Press, London 1980.

[14] W. Jones, N.H. March, Theoretical Solid State Physics, Vol. 2, Dover, New York 1985.

[15] J.P. Šetrajčić, V.M. Zorić, S.M. Vučenović, D.Lj. Mirjanić, V.D. Sajfert, S.K. Jaćimovski, D.I. Ilić, Materials Science Forum 555, 291 (2007).

[16] J.P. Šetrajčić, D.Lj. Mirjanić, S.M. Vučenović, D.I. Ilić, B. Markoski, S.K. Jaćimovski, V.D. Sajfert, V.M. Zorić, Acta Phys. Pol. A 115, 778 (2009).

[17] S.K.Jaćimovki, J.P. Šetrajčić, M.S. Jaćimovski, V.V. Stojanović, Acta Phys. Pol. A 126, 811 (2014).

[18] J.P. Šetrajčić, S.K. Jaćimovski, NBP 20, 67 (2015). ; ISSN 0354-8872

[19] J.P. Šetrajčić, V.D. Sajfert, S.K.Jaćimovski, Rev. Theor. Sci. 4, 353 (2016).

[20] J.P. Šetrajčić, S.K. Jaćimovski, S.M. Vučenović, Academy of Criminalistic, Police Studies, Zemun Belgrade (2017)

[21] B.S. Tošić, J.P. Šetrajčić, R.P. Djajić, D.Lj. Mirjanić, Phys. Rev. B 36, 9094 (1987).

[22] J.P. Šetrajčić, V.M. Zorić, N.V. Delić, D.Lj. Mirjanić, S.K.Jaćimovski, in: Thermodynamics, Ed.: M. Tadashi, InTech, Vienna 2011, Ch. 15, p. 317.

[23] J.P. Šetrajčić, Zaštita materijala 57, 81 (2016). 